\title{
Plasma Zinc Difference in Children with Thalassemia $\beta$ Major Received Deferiprone or Deferasirox Zinc
}

\author{
Wahyu Kusumawardhani, Harsono Salimo, Muhamad Riza \\ Department of Pediatrics, Faculty of Medicine, Universitas Sebelas Maret/ \\ Dr. Moewardi Hospital, Surakarta
}

\section{ABSTRACT}

Background: Thalassemia is a blood disease characterized by the most frequently found autosomal recessive hereditary hemolytic anemia. It requires repeated blood transfusions for life. Routine blood transfusion can cause complications in the form of accumulation of ferritin in the tissue. Iron chelation therapy is considered effective for treating body iron deposits. However, iron chelation therapy has the side effect of decreasing levels of other important minerals such as zinc $(\mathrm{Zn})$. This study aimed to examine plasma zinc difference in children with Thalassemia $\beta$ major received deferiprone or deferasirox zinc.

Subjects and Method: This was a cross sectional study conducted at Dr. Moewardi Hospital, Surakarta, from February to April 2017. A sample of 40 children with thalassemia $\beta$ major aged 3 to 18 years who received deferiprone iron chelation and deferasirox at least 6 months was selected by consecutive sampling. The dependent variable was serum zinc levels. The independent variables were iron deferiprone and deferasirox. Plasma zinc levels were measured by atomic absorption spectroscope. The data were analyzed by t test. Results: Zinc levels in patients with deferiprone therapy $(\mathrm{Mean}=54.50 ; \mathrm{SD}=11.02)$ were lower than deferasirox therapy (Mean $=60.95 ; \mathrm{SD}=$ 20.71), but statistically not significant $(\mathrm{p}=0.229)$. Conclusion:Zinc levels in patients with deferiprone therapy are lower than deferasirox therapy, but not statistically significant.

Keywords: zinc, deferiprone, deferasirox, children with thalassemia $\beta$ major

\section{Correspondence:}

Wahyu Kusumawardhani. Department of Pediatrics, Faculty of Medicine, Universitas Sebelas Maret/ Dr. Moewardi Hospital, Surakarta, Central Java. Phone/ Fax: 0271-633348. Email: dhanisuryadiraja@gmail.com

Cite this as:

Kusumawardhani W, Salimo H, Riza M (2020). Plasma Zinc Difference in Children with Thalassemia $\beta$ Major Received Deferiprone or Deferasirox Zinc. Indones J Med. 05(02): 102-108. https://doi.org/10.26911/theijmed.2020.05.02.02

cc) (7) (-) Indonesian Journal of Medicine is licensed under a Creative Commons Attribution-NonCommercial-ShareAlike 4.0 International License.

\section{BACKGROUND}

Thalassemia is a blood disease characterized by autosomal recessive hereditary hemolytic anemia which is most commonly found (Permono, 2006; David W, 2004). Thalassemia sufferers require lifelong repeated blood transfusions due to hemolytic processes that occur continuously (Permono, 2006; Capellini, 2008, Lanzkowsky P, 2011). Routine blood transfusion can cause accumulation of iron levels in tissues and organs and can affect the function of these organs, including the heart, liver, endocrine glands, bones, lungs and central nervous system (BorgnaPignatti, 2004; Capellini, 2008; Khan, 2007).

To overcome the body's iron deposits, iron chelation therapy is used. The therapy is considered effective in reducing ferritin levels, but has another effect, which is to decrease other important minerals in the body, namely zinc (Satwani, 2005). Existing studies have different results regarding the effect of giving a certain iron chelation to plasma zinc 
Kusumawardhani et al / Plasma zinc difference in children with thalassemia $\beta$ major

levels, other than that there is no consensus on the examination of zinc levels in patients with thalassemia major, making researchers interested in doing about differences in serum zinc levels in patients who get deferiprone iron chelation with deferasirox in thalassemia major $\beta$ patients.

\section{SUBJECTS AND METHOD}

\section{Study Design}

This was an analytic observational study with a cross sectional design.

\section{Population dan Sample}

Subjects in this study were pediatric thalassemia $\beta$ major patients aged 3-18 years. The target population is pediatric patients with thalassemia $\beta$ major. Affordable population is pediatric thalassemia major $\beta$ patients who are in the Hospital Dr. Moewardi Surakarta between February 2017 to April 2017. Sampling as a research subject was conducted by consecutive sampling method. Each pediatric thalassemia $\beta$ major patient who met the study criteria was included in the study. The inclusion criteria were pediatric thalassemia patients aged 3-18 years who were treated between February 2017 and April 2017 with a length of deferiprone and deferasirox iron chelation for a minimum of 6 months, and parents or guardians were willing to sign the research informed consent. Exclusion criteria were patients with poor nutrition, diarrhea, and patients who took zinc preparations within 14 days before sampling.

\section{Study Variables}

The independent variables in this study were flat iron deferiprone and deferasirox. The dependent variable of this study is plasma zinc levels. The confounding variables of this study were adherence to taking iron chelation medication, inadequate nutrition intake, parental education status, socioeconomic status.

\section{Operational Definition of Variables} Flatfoot iron was divided into 2: (1) deferasirox; (2) deferiprone, which is consumed by patients with thalassemia $\beta$ major for at least 1 year at the time of the study, with a dose of $75-100 \mathrm{mg} / \mathrm{kg}$ of deferiprone and a dose of 20-30 mg/ kg of deferiprox.

Plasma zinc levels were measured using an atomic absorption spectroscope (AAS) by taking patients' vein blood in the morning as much as $4 \mathrm{ml}$, were carried out in clinical laboratories, in units of grams/ dl. The measurement scale is continuous and converted to a dichotomy, code $\mathrm{O}=<7 \mathrm{O} \mathrm{g} / \mathrm{dl}$ and nor$\mathrm{mal}=\geq 70 \mathrm{~g} / \mathrm{dl}$.

Age was determined by the year value calculated at the time of data collection. The measurement scale is continuous.

Gender was divided into male and female. The measurement scale is categorical.

Nutritional status was measured using a left upper arm circumference (MUAC) measurement. The circumference of the upper arm is measured at the midpoint of the upper arm between the mid-acromion of the scapula and the olecranon when the hand is flexed at a $90^{\circ}$ angle. The measuring instrument is the medline tape, the unit is in $\mathrm{cm}$, the scale is ordinal, divided into three classes, namely good nutrition, poor nutrition, and poor nutrition.

\section{Data Analysis}

Characteristics of the sample are described in $\mathrm{n}$ and \%. Differences in zinc levels in the deferiprone and deferasirox iron chelation group were analyzed using independent $t$ test.

\section{Research Ethic}

This study was approved by the Board of Health Research Ethics Commission Dr. Moewardi Hospital/ Sebelas Maret University Faculty of Medicine Number: 851/X/ HREC / 2017. 


\section{RESULTS}

\section{A.Sample Characteristics}

Sample characteristics data were described in Table 1.

\section{B. Univariate analysis}

This study was carried out on 40 pediatric Thalassemia $\beta$ major patients where 20 patients were given iron deferasirox chelation therapy and 20 other patients were treated Tabel 1. Sample of Characteristics with iron deferiprone chelation. Characteristic data are in the form of categorical data where the gender is tested differently with Chi Square because nominal categorical data while the number of transfusions and MoryskyAdehernce Scale the Mann Whitney test is done differently because the data with ordinal scale.

\begin{tabular}{|c|c|c|c|c|c|}
\hline \multirow{3}{*}{ Characteristics } & \multicolumn{4}{|c|}{ Flatfoot iron } & \multirow{3}{*}{$\mathbf{p}$} \\
\hline & \multicolumn{2}{|c|}{ Deferasirox } & \multicolumn{2}{|c|}{ Deferiprone } & \\
\hline & $\mathbf{n}$ & $\%$ & $\mathbf{N}$ & $\%$ & \\
\hline \multicolumn{6}{|l|}{ Gender } \\
\hline Male & 12 & $60.0 \%$ & 6 & $30.0 \%$ & 0.057 \\
\hline Female & 8 & $40.0 \%$ & 14 & $70.0 \%$ & \\
\hline \multicolumn{6}{|l|}{ Amount of Transfusion } \\
\hline 1 bags & 8 & $40.0 \%$ & 4 & $20.0 \%$ & 0.163 \\
\hline 2 bags & 11 & $55.0 \%$ & 14 & $70.0 \%$ & \\
\hline 3 bags & 1 & $5.0 \%$ & 2 & $10.0 \%$ & \\
\hline Morysky Adeherence Scale & & & & & 0.009 \\
\hline O & 1 & $5.0 \%$ & $\mathrm{O}$ & $\mathrm{O} \%$ & \\
\hline 1 & 5 & $25.0 \%$ & $\mathrm{O}$ & $0 \%$ & \\
\hline 2 & 14 & $70.0 \%$ & 20 & $100.0 \%$ & \\
\hline
\end{tabular}

\section{The result of bivariate analysis}

Table 2 shows that the level of GDS in patients on deferasirox therapy (Mean $=112.90$; $\mathrm{SD}=34.50$ ), higher than deferiprone therapy (Mean $=85.25 ; \mathrm{SD}=30.26)$, and and statistically significant $(\mathrm{p}=0.007)$. SGOT levels in patients on deferasirox therapy (Mean= 100.50; $\mathrm{SD}=68.37)$ were higher than deferiprone therapy $($ Mean $=39.80 ; \mathrm{SD}=10.55)$, and statistically significant $(\mathrm{p}=0.001)$. SGPT levels in patients on deferasirox therapy (Mean $=60.50 ; \mathrm{SD}=52.13$ ) were higher than deferiprone therapy (Mean $=30.95 ; \mathrm{SD}=$ 20.13), and statistically significant $(\mathrm{p}=$ o.o03). Ferritin levels in patients with deferasirox therapy (Mean $=4,105.01 ; \mathrm{SD}=$ 1,394.70) were higher than deferiprone therapy (Mean=4,080.19; $\mathrm{SD}=2,004.34)$, but not statistically significant $(\mathrm{p}=0.964)$.

Table 2. Differences in Lab Results by Type of Deferasirox Flatfoot Therapy and Deferiprone Therapy

\begin{tabular}{|c|c|c|c|c|c|}
\hline \multirow{3}{*}{ Lab results } & \multicolumn{4}{|c|}{ Flatfoot Iron } & \multirow{3}{*}{$\mathbf{p}$} \\
\hline & \multicolumn{2}{|c|}{ Deferasirox } & \multicolumn{2}{|c|}{ Deferiprone } & \\
\hline & Mean & SD & Mean & SD & \\
\hline GDS & 112.90 & 34.50 & 85.25 & 30.26 & 0.007 \\
\hline SGOT & 100.50 & 68.37 & 39.80 & 10.55 & 0.001 \\
\hline SGPT & 60.50 & 52.13 & 30.95 & 20.13 & 0.003 \\
\hline Feritin & 4105.01 & 1394.70 & 4080.19 & 2004.34 & 0.964 \\
\hline
\end{tabular}


Kusumawardhani et al / Plasma zinc difference in children with thalassemia $\beta$ major

Table 3. Differences in zinc levels by type of Deferasirox iron chelation therapy with Deferiprone therapy

\begin{tabular}{lccc}
\hline Flatfoot Iron & Mean & SD & p \\
\hline Deferasirox & 54.50 & 60.95 & 0.229 \\
Deferiprone & 11.02 & 20.71 & \\
\hline
\end{tabular}

Table 3 shows that zinc levels in patients on deferasirox therapy (Mean $=54.50$; $\mathrm{SD}=11.02)$ are lower than deferiprone therapy $($ Mean $=60.95 ; \mathrm{SD}=20.71)$, but statistically not significant $(\mathrm{p}=0.229)$.

Table 4. Differences in Zinc Levels Based on Iron Flatfoot and Blood Transfusion

\begin{tabular}{ccccc}
\hline Flatfoot And Transfusion & n & Mean & SD & p \\
\hline Deferasirox-1 bag & 8 & 56.13 & 13.86 & 0.722 \\
Deferasirox-2bags & 11 & 53.00 & 9.51 & \\
Deferasirox-3bags & 1 & 58.00 & $<0.01$ & \\
Deferasirox-1 bag & 4 & 65.25 & 15.35 & \\
Deferiprone-2bags & 14 & 61.29 & 23.36 & \\
Deferiprone-3bags & 2 & 50.00 & 5.66 & \\
\hline
\end{tabular}

Table 5. Post Hoc Test

\begin{tabular}{llll}
\hline \multicolumn{1}{c}{ Flatfoot And Transfusion } & \multicolumn{1}{c}{ Mean (95\% CI) } & p \\
\hline Deferasirox-1 bag & Deferasirox-2 bags & $3.13(-8.15-14.40)$ & 0.869 \\
Deferasirox-1 bag & Deferasirox-3 bags & $-1.88(-36.64-32.89)$ & 0.699 \\
Deferasirox-1 bag & Deferiprone-1 bag & $-9.13(-28.67-10.42)$ & 0.349 \\
Deferasirox-1 bag & Deferiprone-2 bags & $-5.16(-24.15-13.83)$ & 0.608 \\
Deferasirox-1 bag & Deferiprone-3 bags & $6.13(-17.79-30.04)$ & 0.794 \\
Deferasirox-2 bags & Deferasirox-3 bags & $-5.00(-27.13-17.13)$ & 0.244 \\
Deferasirox-2 bags & Deferiprone-1 bag & $-12.25(-26.29-1.79)$ & 0.102 \\
Deferasirox-2 bags & Deferiprone-2 bags & $-8.29(-23.83-7.26)$ & 0.459 \\
Deferasirox-2 bags & Deferiprone-3 bags & $3.00(-12.61-18.61)$ & 0.843 \\
Deferasirox-3 bags & Deferiprone-1 bag & $-7.25(-61.86-47.36)$ & 0.480 \\
Deferasirox-3 bags & Deferiprone-2 bags & $-3.29(-55.53-48.96)$ & 0.643 \\
Deferasirox-3 bags & Deferiprone-3 bags & $8.00(-80.03-96.03)$ & 0.221 \\
Deferiprone-1 bag & Deferiprone-2 bags & $3.96(-22.58-30.51)$ & 0.671 \\
Deferiprone-1 bag & Deferiprone-3 bags & $15.25(-17.43-47.93)$ & 0.165 \\
Deferiprone-2 bags & Deferiprone-3 bags & $11.29(-25.30-47.87)$ & 0.577 \\
\hline
\end{tabular}

Table 6. Differences in zinc levels based on nutritional status

\begin{tabular}{cccc}
\hline Nutritional Status & Mean & SD & p \\
\hline Poor & 54.29 & 16.59 & 0.180 \\
Good & 60.26 & 16.68 & \\
\hline
\end{tabular}

Table 6 shows zinc levels in patients with good nutritional status (Mean=60.26; $\mathrm{SD}=$ 16.68) higher than poor nutritional status
(Mean= 54.29; $\mathrm{SD}=16.59)$ but statistically not significant $(\mathrm{p}=0.180)$. 


\section{DISCUSSION}

1. Sample characteristic in children with thalassemia $\beta$ Major

Characteristic data showed that there were no significant differences ( $\mathrm{p}>0.05$ ) in sex and number of blood transfusions in the Deferasirox and Deferiprone iron chelers. This shows that the distribution of samples was evenly distributed across all sex groups and the amount of blood transfusion. All Deferiprone Iron Flatfoot respondents and most (70\%) Deferasirox Flatfoot respondents have been undergoing treatment routinely based on the Morysky Adherence Scale score. However, there are significant differences between the two groups, so this is thought to be able to influence the results of the next follow-up examination. According to an article, non-compliance with thalassemia patients in implementing chelation therapy programs can be caused by psychological disorders in patients, relatives with similar diseases, low family economy, lack of parental supervision, and reduced frequency of blood transfusions (Al-Kloub et al, 2014). Another article also agreed to explain that this non-compliance is caused by the patient's psychological condition, which is coupled with the patient's behavioral factors, such as living alone and difficult access to treatment (Vosper et al, 2018).

\section{The laboratory tests in children with} thalassemia $\beta$ Major

Laboratory tests show that individuals treated with Deferasirox will have a significantly higher GDS, SGOT, and SGPT than individuals with Deferiprone therapy. Based on several articles, the increase in SGOT and SGPT is related to liver damage due to iron overload due to blood transfusion, and is not related to administration of iron chelation therapy (Jensen, 2002; Hagag et al., 2015). This difference shows that administration of Deferiprone has a therapeutic effect on liver damage due to iron overload better than
Deferasirox. On the other hand, normal and relatively low mean GDS values in respondents after iron chelation therapy were also found in several other studies (Chuansumrit et al., 2016; Gomber et al., 2017).

According to both studies, the administration of iron chelation will reduce the iron build up in the pancreas, thus improving the workings of pancreatic beta cells. In addition, Chuansumrit et al also suspected that insulin sensitivity would improve with administration of iron chelation in beta-type thalassemia patients. From these results, it appears that Deferiprone has a therapeutic effect on pancreatic damage due to iron overlaod better than Deferasirox. The absence of a significant difference between Deferiprone and Deferasirox against serum ferritin shows that both iron sailors have an equally strong effect in regulating serum ferritin levels.

\section{The results of examination of zinc in children with thalassemia $\beta$ Major}

The results of examination of zinc levels in patients did not show significantly different results in administration of Deferasiroxchelets and Deferipronechelers. This is in accordance with some previous studies (Lachowicz et al, 2015; Zekavat et al, 2018).

Zekavat et al. (2018), shows that there is no significant difference between variations of the type of iron chelation drug given to patients against zinc levels in patients. The same study also mentioned that the group that took iron chelation therapy had lower serum zinc levels than those who were not treated with iron chelation. The administration of iron chelation agents is thought to interfere with homeostasis from the equilibrium of zinc (II) ion bonds, thereby causing a decrease in serum zinc levels (Lachowicz et al., 2015). Based on these results, Deferasirox flatfoot and Deferiprone flatfoot have the same strong effect in reducing serum zinc levels. 
Kusumawardhani et al / Plasma zinc difference in children with thalassemia $\beta$ major

Zinc levels did not differ significantly with each transfusion bag and iron chelation. A study states that the administration of blood transfusion can significantly increase serum zinc levels (Kizilgun et al., 2016). This is thought to be caused by the effect of Deferasirox and Deferiprone iron chelating agents which are equally strong in reducing zinc levels in patients, so the differences between groups become insignificant (Zekavat et al, 2018). However, it is suspected there are other reasons that cause this insignificant difference. First, the distribution of respondents was uneven in several groups. Each group does not consist of the same number of respondents, so it will affect the average of each group. Secondly, the presence of zinc intake in the respondents' diet is thought to have influenced the results of serum zinc levels. Respondents with lower zinc consumption will have lower serum zinc levels, and are more at risk of developing growth disorders during the course of thalassemia (Fung, 2016).

\section{The nutritional status in children with thalassemia $\beta$ Major}

The results showed that nutritional status was not significantly related to serum zinc levels in patients. However, based on several existing studies, there is a relationship between nutritional status and serum zinc levels (Fung, 2016; Kulathinal et al., 2016). This is likely because the nutritional status of the patient is measured by measuring the upper arm circumference. A study states that the measurement of nutritional status by the waist circumference method shows a significant relationship to serum zinc levels in respondents (Kulathinal et al, 2016).

Limitation of this study is the absence of respondents who act as controls in the study. The control meant were respondents with beta thalassemia who did not undergo blood transfusion and iron chelation therapy, or who did blood transfusion but were not treated with iron chelation. A study with pre and post test evaluation methods is also needed to be able to evaluate differences in laboratory value parameters such as zinc, ferritin, GDS, SGOT, SGPT before and after iron chelation is given.

Based on the results, it can be concluded that there is an influence between the administration of iron chelation on zinc, GDS, SGOT, SGPT, and ferritin levels. There are also significant differences between Ferrosiprox and Deferasirox chelation on serum levels of GDS, SGOT, and SGPT, but not on zinc and ferritin levels. In addition, the patient's nutritional status is not related to zinc levels in the patient.

\section{AUTHOR CONTRIBUTION}

Wahyu Kusumawardhani was the main author in this study, measured plasma zinc level, wrote the manuscript. Harsono Salimo and Muhamad Riza interpreted the results of study.

\section{CONFLICT OF INTEREST}

There was no conflict of interest in this study.

\section{FUNDING AND SPONSORSHIP}

This study was self-funded

\section{ACKNOWLEDGEMENT}

The author would like to thank all those who have helped this research, to the patients and parents of the study subjects, Head of Room and Nurse of Melati 2 Room, Clinical Pathology Laboratory Dr. Moewardi Surakarta, and all those who helped in this research.

\section{REFERENCE}

Hagag AA, Elfaragy MS, Elrifaey SM, Abd ElLateef AE (2015). Therapeutic value of combined therapy with deferiprone and silymarinas iron chelators in Egyptian children with beta thalassemia major. Infect Disord Drug Targets, 
Kusumawardhani et al / Plasma zinc difference in children with thalassemia $\beta$ major

15(3): 189 - 195. https://doi.org/ 10.2174/1871526515666150731113305

Al-Kloub MI, A Bed MA, Al Khawaldeh OA, Al Tawarah YM, Froelicher ES (2014). Predictors of non-adherence to followup visits and deferasirox chelation therapy among Jordanian adolescents with thalassemia major. Pediatr Hematol Oncol, 31(7): 624-637. https://doi.org/10.3109/08880018.2014.939792

Chuansumrit A, Pengpis P, Mahachoklertwattana P, Sirachainan N, Poomthavorn P, Sungkarat W, Kadegasem P, Khlairit P, Wongwerawattanakoon P (2016). Effect of iron chelation therapy on glucose metabolism in non-transfusion dependent thalassaemia. Acta Haematol, 137(1): 20-26. https://doi.org/10.1159/000450673

Fung EB (2016). The importance of nutrition for health in patients with transfusiondependent thalassemia. Ann $\mathrm{Ny}$ Acad Sci, 1368(1): 40-48. https://doi.org/10.1111/nyas.13003

Gomber S, Dabas A, Bagmar S, Madhu SV (2017). Glucose homeostasis and effect of chelation on $\beta$ cell function in children with $\beta$-Thalassemia major. J Pediatr Hematol Oncol. 40(1):56-59. https://doi.org/10.1097/MPH.ooooo00000001043

Jensen PD (2002). Relationship between hepatocellular injury and transfusional iron overload prior to and during iron chelation with desferrioxamine: a study in adult patients with acquired anemias. Bld, 101(1): 91-96. https://doi.org/10.1182/blood-2002-06-1704
Kizilgun M, Takci S, Erkekoglu P, Asci A, Balci A, Yigit S, Kocer-Gumusel B (2016). Copper, zinc and iron levels in premature infants following red blood cell transfusion. J Trace Elem Med Bio, 38: 126-130. https://doi.org/10.1016/j.jtemb.2016.05.011

Kulathinal S, Freese R, Korkalo L, Ismael C, Mutanen M (2016). Mid-upper arm circumference is associated with biochemically determined nutritional status indicators among adolescent girls in Central Mozambique. Nutr Res Rev, 36(8): 835-844. https://doi.org/10.1016/j.nutres.2016.04.007

LachowiczJI, NurchiVM, Crisponi G, Jaraquemada-Pelaez MG, Ostrowska M, Jezierska J, Gumienna-Kontecka E, et al. (2015). Zinc(II) and copper(II) complexes with hydroxypyrone iron chelators. J Inorg Biochem, 151: 94-106. https://doi.org/10.1016/j.jinorgbio.2015.08.011

Vosper J, Evangeli M, Porter JB, Shah F (2018). Psychological Factors Associated with Episodic Chelation Adherence in Thalassemia. Hb, 42(1): 30-36. https://doi.org/10.1080/03630269.2018.1433686

Zekavat OR, Bahmanjahromi A, Haghpanah S, Ebrahimi S, Cohan N (2018). The Zinc and Copper Levels in Thalassemia Major Patients, Receiving Iron Chelation Therapy. J Pediatr Hematol Oncol. 40(3): 178-181. https://doi.org/10.1097/mph.0000000000001102 